\title{
ARTICLE OPEN \\ Mechanochemical spinodal decomposition: a phenomenological theory of phase transformations in multi-component, crystalline solids
}

\author{
Shiva Rudraraju', Anton Van der Ven ${ }^{2}$ and Krishna Garikipati ${ }^{3,4}$
}

We present a phenomenological treatment of diffusion-driven martensitic phase transformations in multi-component crystalline solids that arise from non-convex free energies in mechanical and chemical variables. The treatment describes diffusional phase transformations that are accompanied by symmetry-breaking structural changes of the crystal unit cell and reveals the importance of a mechanochemical spinodal, defined as the region in strain-composition space, where the free-energy density function is non-convex. The approach is relevant to phase transformations wherein the structural order parameters can be expressed as linear combinations of strains relative to a high-symmetry reference crystal. The governing equations describing mechanochemical spinodal decomposition are variationally derived from a free-energy density function that accounts for interfacial energy via gradients of the rapidly varying strain and composition fields. A robust computational framework for treating the coupled, higher-order diffusion and nonlinear strain gradient elasticity problems is presented. Because the local strains in an inhomogeneous, transforming microstructure can be finite, the elasticity problem must account for geometric nonlinearity. An evaluation of available experimental phase diagrams and first-principles free energies suggests that mechanochemical spinodal decomposition should occur in metal hydrides such as $\mathrm{ZrH}_{2-2 c}$. The rich physics that ensues is explored in several numerical examples in two and three dimensions, and the relevance of the mechanism is discussed in the context of important electrode materials for Li-ion batteries and high-temperature ceramics.

npj Computational Materials (2016) 2, 16012; doi:10.1038/npjcompumats.2016.12; published online 10 June 2016

\section{INTRODUCTION}

Spinodal decomposition is a continuous phase transformation mechanism occurring throughout a solid that is far enough from the equilibrium for its free-energy density to lose convexity with respect to an internal degree of freedom. The latter could include the local composition as in classical spinodal decomposition described by Cahn and Hilliard, ${ }^{1}$ or a suitable non-conserved order parameter as in the theory by Allen and $\mathrm{Cahn}^{2}$ for spinodal ordering. A key requirement for continuous transformations is that order parameters can be formulated to uniquely describe continuous paths connecting the various phases of the transformation. These phases then correspond to local minima on a single, continuous free-energy density surface in that order parameter space. For classical spinodal decomposition inside a miscibility gap, all phases have the same crystal structure and symmetry, and the order parameter is simply the local composition. The existence of a single, continuous free-energy density surface for all phases participating in a transformation implies, by geometric necessity, the presence of domains in order parameter space, where the free-energy density is non-convex. Reaching those domains through supersaturation (by externally varying temperature or composition) makes the solid susceptible to a generalised spinodal decomposition.

Many important multi-component solids undergo phase transformations that couple diffusional redistribution of their components with a structural change of the crystallographic unit cell. One prominent example is the decomposition that occurs when cubic yttria-stabilised zirconia $\mathrm{Zr}_{1}{ }_{-} \mathrm{Y}_{c} \mathrm{O}_{2-c / 2}$ is quenched into a two-phase equilibrium region between tetragonal $\mathrm{Zr}_{1}-{ }_{c} \mathrm{Y}_{c} \mathrm{O}_{2-c / 2}$ having a low-Y composition and cubic $\mathrm{Zr}_{1}-{ }_{c} \mathrm{Y}_{c} \mathrm{O}_{2-c / 2}$ having a high-Y composition. Another occurs in Li-battery electrodes made of spinel $\mathrm{Li}_{c} \mathrm{Mn}_{2} \mathrm{O}_{4}$. Discharging to low voltages causes the compound to transform from cubic $\mathrm{LiMn}_{2} \mathrm{O}_{4}$ to tetragonal $\mathrm{Li}_{2} \mathrm{Mn}_{2} \mathrm{O}_{4}$ through a two-phase diffusional phase transformation mechanism. As with simple diffusional phase transformations, these coupled diffusional/martensitic phase transformations can occur either through a nucleation and growth mechanism or, if certain symmetry requirements are met, through a continuous mechanism due to an onset of an instability with respect to the composition and/or a structural order parameter.

Here we present a treatment of coupled diffusional/martensitic phase transformations triggered by instabilities with respect to both strain and composition. These phase transformations are characterised by a mechanochemical spinodal that is defined as a non-convex region of the free-energy density function in the strain-composition space. The possibility of a mechanochemical spinodal decomposition is motivated by the recent first-principles studies of martensitic transformations in transition metal hydrides, where a high-temperature cubic phase is predicted to display negative curvatures with respect to strain, thus making strain a natural order parameter to distinguish the cubic parent phase

${ }^{1}$ Department of Mechanical Engineering, University of Michigan, Ann Arbor, MI, USA; ${ }^{2}$ Materials Department, University of California, Santa Barbara, CA, USA; ${ }^{3}$ Department of Mechanical Engineering, University of Michigan, Ann Arbor, MI, USA and ${ }^{4}$ Department of Mathematics, University of Michigan, Ann Arbor, MI, USA.

Correspondence: K Garikipati (krishna@umich.edu)

Received 17 January 2016; revised 26 April 2016; accepted 5 May 2016 
from its low-temperature tetragonal daughter phases. ${ }^{3}$ In addition, the coupling with composition degrees of freedom (e.g., through the introduction of hydrogen vacancies in the metal hydrides) allows for the possibility that the free energy also exhibits a negative curvature with respect to the composition. Mechanochemical spinodal decomposition, therefore, is a phenomenon that is likely present in many multi-component materials but has to date been overlooked as a mechanism by which a high-symmetry phase can decompose martensitically and through diffusional redistribution upon quenching into a two-phase regime. Structural phase transformations in solids driven by instability with respect to an internal shuffle of the atoms within the unit cell have been treated rigorously in the literature with coupled Cahn-Hilliard and Allen-Cahn approaches, ${ }^{4-7}$ but mechanochemical spinodal decomposition is fundamentally different and necessitates a coupled treatment of both the strain and composition instabilities.

Our treatment is based on a generalised, Landau-type free-energy density function that couples strain and composition instabilities. The governing equations of mechanochemical spinodal decomposition, obtained by variational principles, generalise the classical equations of the Cahn-Hilliard formulation, ${ }^{1}$ and of nonlinear gradient elasticity ${ }^{8,9}$ by coupling these systems. The ability to solve this complex, nonlinear, strainand composition-gradient-driven, mechanochemical system for sufficiently general initial and boundary value problems in two and three dimensions also has been lacking heretofore. We introduce the computational framework to obtain such solutions in general, three-dimensional solids. This new-found capability allows us to then reinforce our discussion of the phenomenology with dynamics predicted by the numerical solutions.

The mechanochemical spinodal in two dimensions

For accessibility of the arguments, we first consider the twodimensional analogue of the cubic-to-tetragonal transformation: the square-to-rectangle transformation. The high-symmetry square lattice will serve as the reference crystal relative to which strains are measured. Lower-symmetry lattices that can be derived from the square lattice by homogeneous strain include the rectangle, the diamond and lattices, where there are no constraints on the cell lengths and their angles.

We use the composition $c$, varying between 0 and 1 , as our order parameter for the chemistry of our binary two-dimensional solid. Symmetry-breaking structural changes are naturally described by the strain relative to the high-symmetry square lattice. The elastic free-energy density is also a function of strain. In both contexts, strain will in general be of finite magnitude. Following Barsch and Krumhansl, ${ }^{8}$ we use the Green-Lagrange strain tensor, $\boldsymbol{E}$, relative to the square lattice. Rotations are exactly neutralised in this strain measure; for any rigid body motion, $\boldsymbol{E}=0$ (Supplementary Information). In two dimensions, the relevant strain components are $E_{11}, E_{22}$ and $E_{12}=E_{21}$. However, it is more convenient to use linear combinations of these components that transform according to the irreducible representations of the point group of the high-symmetry square lattice. In two dimensions, these include $e_{1}=\left(E_{11}+E_{22}\right) \sqrt{2}, \quad e_{2}=\left(E_{11}-E_{22}\right) \sqrt{2}$ and $e_{6}=\sqrt{2} E_{12}$. Here $e_{1}$ and $e_{6}$ reduce to the dilatation and shear strain, respectively, in the infinitesimal strain limit. The strain measure $e_{2}$ uniquely maps the square lattice into the two rectangular variants (Figure 1a): positive and negative $e_{2}$ generate the rectangles elongated along the global $X_{1}$ and $X_{2}$ directions, respectively. The equivalence of the rectangular variants under the point group symmetry of the square lattice $\left(e_{2}=0\right)$ restricts the free-energy density to even functions of $e_{2}$.

If the crystalline solid has multiple chemical species, its free-energy density dependence on $e_{1}, e_{2}$ and $e_{6}$ can change with composition, c. Figure $2 a$ illustrates a free-energy density
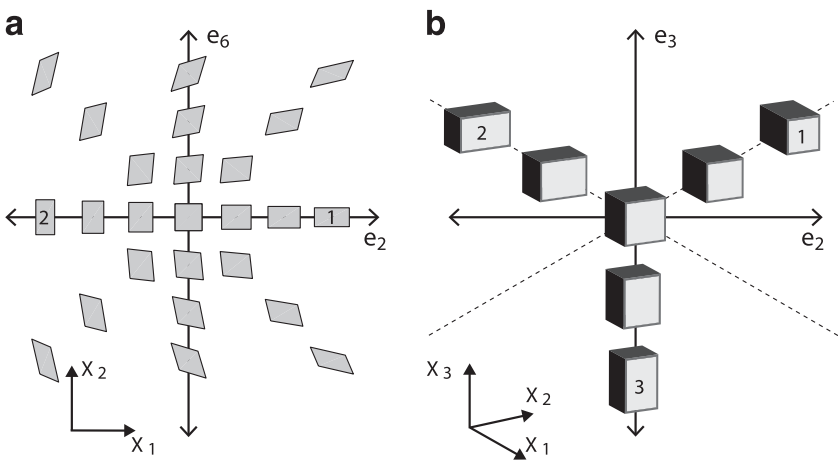

Figure 1. (a) Square-to-rectangle transition (2D) in reparametrised strain space. (b) Cubic-to-tetragonal transition (3D) in reparametrised strain space (equation 1). Lattice vectors are labelled by the corresponding coordinate directions $x_{1}, x_{2}$ and $x_{3}$, and the corresponding lower-symmetry phases are labelled 1, 2 and 3 . The deformations shown in the sub-figures are area/volume preserving, respectively.
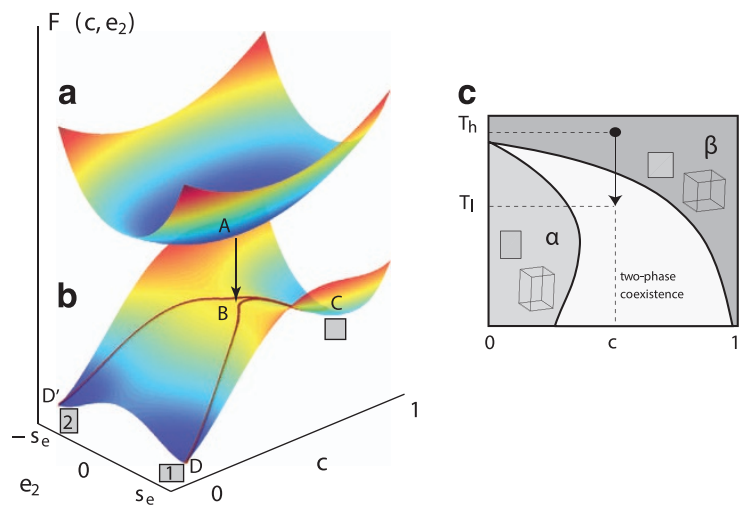

Figure 2. (a) Free-energy density for the $2 \mathrm{D}$ formulation at high temperature and (b) at low temperature, showing the mechanochemical spinodal along with two energy-minimising paths (brown lines) and their corresponding minimum energy-strained structures. (c) Temperature-composition phase diagram.

surface, $\mathscr{F}\left(c, e_{2}\right)$, for a binary solid that, at higher temperature, forms a solid solution having square symmetry. In this case, $\mathscr{F}$ is convex, a condition made precise by specifying positive eigenvalues of its Hessian matrix over the $\left\{c, e_{2}\right\}$ space. In addition, $\mathscr{F}(c, 0)$ is a minimum with respect to $e_{2}$ for fixed $c$, making the square phase stable for all $c$ at this temperature. However, at a lower temperature, $\mathscr{F}$ may lose convexity, inducing the notion of a mechanochemical spinodal region. We define it as the domain in $\left\{c, e_{2}\right\}$ space over which the Hessian matrix admits negative eigenvalues, as illustrated in Figure $2 \mathrm{~b}$. Here we focus on the conditions $\partial^{2} \mathscr{F} / \partial c^{2}<0$ and $\partial^{2} \mathscr{F} / \partial e_{2}^{2}<0$. The square phase $\left(e_{2}=0\right)$ remains stable at high composition $(c \sim 1)$ with positive eigenvalues of the Hessian (Figure 2b). But it is mechanically unstable at low composition, with $\partial^{2} \mathscr{F} / \partial e_{2}^{2}<0$ for $\left(c, e_{2}\right) \sim(0,0)$, and has two symmetrically equivalent, stable, rectangular phases, with $\partial^{2} \mathscr{F} / \partial e_{2}^{2}>0$ at $\left(c, e_{2}\right)=\left(0, \pm s_{e}\right)$. Although not shown in Figure $2 \mathrm{~b}$, we assume that $\mathscr{F}$ is convex with respect to $e_{1}$ and $e_{6}$. Figure $2 c$ illustrates a schematic temperature-composition phase diagram consistent with the free-energy densities of Figure $2 a, b$. The square/cubic phase, $\beta$, forms at high composition or at high temperature; the rectangle/tetragonal phase, $a$, forms at low composition and low temperature. A large two-phase region separates them.

Zuzek et al. ${ }^{10}$ have obtained phase diagrams experimentally that are topologically equivalent to Figure $2 c$ for the $\mathrm{ZrH}_{2-2 c}$ system. Recent first-principles calculations ${ }^{3}$ have demonstrated 
the existence of a mechanical instability that exists in this system at low $c$ via non-convexity with respect to strain. Furthermore, the two-phase coexistence at low temperature also implies non-convexity with respect to the composition, as we have demonstrated in Supplementary Information. Figure $2 \mathrm{a}, \mathrm{b}$ therefore represents a two-dimensional analogue of the free energy for such systems.

Consider our model binary solid, annealed at high temperature $T_{h}$, to form a solid solution in the square phase, $\beta$. Its state is at point $\mathrm{A}$ in Figure $2 \mathrm{a}$, with $e_{2}=0$. It is then quenched into the two-phase region (Figure $2 \mathrm{c}$ ) with free-energy density at point $B$ in Figure $2 \mathrm{~b}$. For a quench at sufficiently high rate, the dimensions of the square lattice, controlled by strains $e_{1}, e_{2}$ and $e_{6}$, and the composition remain momentarily unchanged. However, as the state at point $B$ satisfies $\partial^{2} \mathscr{F} / \partial e_{2}^{2}<0$ and $\partial^{2} \mathscr{F} / \partial c^{2}<0$, there exist thermodynamic driving forces for segregation by strain and composition within the mechanochemical spinodal.

Diffusion being substantially slower than elastic relaxation, the solid immediately deforms to either positive or negative $e_{2}$, driven towards a local minimum at constant, $c$. These deformations due to the mechanical instability will happen like many martensitic transformations, where a mix of symmetrically equivalent rectangular variants coexist to minimise macroscopic strain energy. For finite but moderate strain, the transformation could proceed coherently, ${ }^{11}$ even if the two symmetrically equivalent rectangular variants coexist. We neglect non-essential complexities of this process and assume that, instantly upon quenching, finitely sized neighbourhoods of the solid deform homogeneously into one of these rectangular variants at the original composition.

The solid also becomes susceptible to uphill diffusion because $\partial^{2} \mathscr{F} / \partial c^{2}<0$ implies a negative diffusion coefficient. However, it does not occur at constant $e_{2}$, as the valleys traversing the local minima, $\partial \mathscr{F} / \partial e_{2}=0$, between the square lattice at $c=1$ and the rectangular lattices at $c=0$ span intervals of negative and positive $e_{2}$. Mechanochemical spinodal decomposition sets in. Composition modulations are amplified: high-c regions strive to be more square (point $C$ ), whereas low- $c$ regions strive to be more rectangular (points $\mathrm{D}$ or $\mathrm{D}^{\prime}$ ). However, as coherency is maintained, some neighbourhoods in the solid will be frustrated from attaining strains that ensure minima, $\partial \mathscr{F} / \partial e_{2}=0$, for local values of $c$. Coherency strain-induced free-energy penalties arise to alter the driving forces for purely chemical spinodal decomposition. Supplementary Movie S5 in the Supplementary Information shows the evolution of the state $\left(c, e_{2}\right)$ of the material points on the free-energy manifold $\mathscr{F}$.

\section{Mathematical formulation: three dimensions}

Armed with the insight conveyed by the two-dimensional study, we next lay out the three-dimensional treatment, in which setting the considered phase transformations proceed via lattice deformation and diffusion. The arguments are made more concrete by considering the general mathematical form of the free-energy density.

Strain order parameters. The strain measures $e_{1}-e_{6}$ are first redefined using the full Green-Lagrange strain tensor components in three dimensions:

$$
\begin{aligned}
& e_{1}=\frac{1}{\sqrt{3}}\left(E_{11}+E_{22}+E_{33}\right), e_{2}=\frac{1}{\sqrt{2}}\left(E_{11}-E_{22}\right), \\
& e_{3}=\frac{1}{\sqrt{6}}\left(E_{11}+E_{22}-2 E_{33}\right), e_{4}=\sqrt{2} E_{23}=\sqrt{2} E_{32}, \\
& e_{5}=\sqrt{2} E_{13}=\sqrt{2} E_{31}, e_{6}=\sqrt{2} E_{12}=\sqrt{2} E_{21}
\end{aligned}
$$

In the limit of infinitesimal strains, $e_{1}$ describes the dilatation, whereas $e_{4}, e_{5}$ and $e_{6}$ reduce to shears. The point group operations of the cubic crystal leave $e_{1}$ invariant, as it is the trace of $\boldsymbol{E}$, while collecting each of the subsets $\left\{e_{2}, e_{3}\right\}$ and $\left\{e_{4}, e_{5}, e_{6}\right\}$ into a symmetry-invariant subspace whose elements transform into each

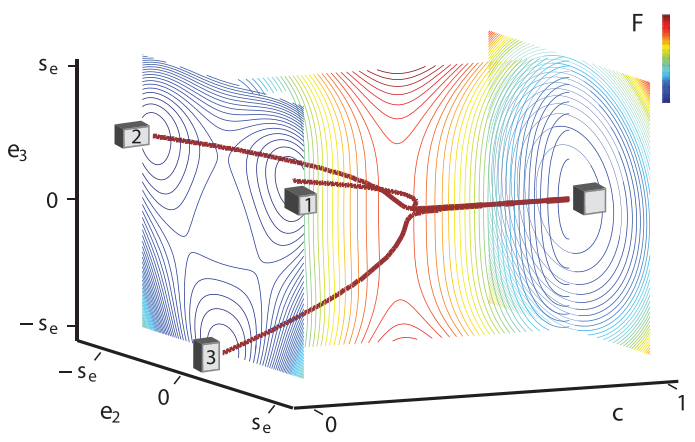

Figure 3. Mechanochemical spinodal for the 3D formulation depicted by contours of the free-energy manifold along the $e_{2}-e_{3}$ and $e_{3}-c$ planes. The three energy-minimising paths (brown lines) and their corresponding energy-minimising-strained structures are also shown.

other. The measures $e_{2}$ and $e_{3}$ are especially suited as order parameters to describe cubic-to-tetragonal distortions. All three tetragonal variants that emerge from the cubic reference crystal can be uniquely represented by these two measures. See Figure $1 b$, where tetragonal distortions of the cubic crystal along the $X_{1}, X_{2}$ and $X_{3}$ axes have been labelled as 1,2 and 3 , respectively. Deviations from the dashed lines in the $e_{2}-e_{3}$ space of Figure $1 \mathrm{~b}$ correspond to orthorhombic distortions of the cubic reference crystal. This role of $e_{2}$ and $e_{3}$ as structural order parameters to denote the degree of tetragonality, and to distinguish between the three tetragonal variants, complements their fundamental purpose as arguments of the elastic free-energy density.

The mechanochemical spinodal in three dimensions. For brevity, we write the strains as $\boldsymbol{e}=\left\{e_{1}, \ldots, e_{6}\right\}$. We introduce further phenomenology by specifying that $\mathscr{F}(c, \boldsymbol{e})$ is only one part of the total free-energy density. It is a homogeneous contribution whose composition and strain dependence cannot be additively separated. Figure 3, for example, illustrates contour plots of $\mathscr{F}(c, \boldsymbol{e})$ on the $e_{2}-e_{3}$ and $e_{3}-c$ planes for a binary solid having a temperature-composition phase diagram similar to that of Figure 2c. To generate the tetragonal a phase as illustrated in that diagram, the homogeneous free-energy density as a function of strain must qualitatively follow the contours of the $e_{2}-e_{3}$ plane at $c=0$ in Figure 3 . Here the tetragonal variants are local minimisers of $\mathscr{F}$ in $e_{2}-e_{3}$ space, with equal minima. In turn, to obtain the cubic $\beta$ phase, $\mathscr{F}(c, \boldsymbol{e})$ must follow the contours of the $e_{2}-e_{3}$ plane at $c=1$. Thus, $\mathscr{F}$ changes smoothly from convex with respect to $e_{2}$ and $e_{3}$ on the $c=1$ plane to non-convex at $c=0$ to form the three variants of the tetragonal $a$ phase. Importantly, the planes at $c=0$ and $c=1$ themselves must be minimum energy surfaces to represent the tetragonal $\alpha$ and cubic $\beta$ phases, respectively. Supplementary Movie S8 in the Supplementary Information shows the evolution of the state $\left(c, e_{2}, e_{3}\right)$ of the material points on the free-energy manifold $\mathscr{F}$.

Gradient regularisation of the free-energy density: Following van der Waals, ${ }^{12}$ and Cahn and Hilliard ${ }^{1}$ in their treatment of nonuniform composition fields, we can extend the total free-energy density beyond $\mathscr{F}$ by writing it as a Taylor series, retaining terms that depend on the composition gradient, $\nabla c$. We extend this gradient dependence to the strain measures $\nabla \boldsymbol{e}$, as did Barsch and Krumhansl $^{8}$ following Toupin. ${ }^{9}$ (Also see Karatha et al., ${ }^{13,14}$ for treatments using infinitesimal and finite strain, respectively.) These gradient dependences appear in a non-uniform free-energy density $\mathscr{G}(c, \boldsymbol{e}, \nabla c, \nabla \boldsymbol{e})$. Frame invariance of $\mathscr{F}$ and $\mathscr{G}$ is guaranteed, as the members of $\boldsymbol{e}$ are linear combinations of the tensor components of $\boldsymbol{E}$. They also must be invariant under point group operations of the cubic reference crystal. 
Free-energy functional. The crystal occupies a reference (undeformed) configuration $\Omega$ with boundary $\Gamma$. The total freeenergy, $\Pi$, is the integral of the free-energy density $\mathscr{F}+\mathscr{G}$ over the solid with boundary contributions included. Thus, $\Pi$ is a functional of the composition $c$ and the displacement vector field $\boldsymbol{u}$, from which the strains are derived (Supplementary Information):

$$
\prod[c, \boldsymbol{u}]=\int_{\Omega}(\mathscr{F}+\mathscr{G}) \mathrm{d} V-\sum_{i=1}^{3} \int_{\Gamma_{T_{i}}} u_{i} T_{i} \mathrm{~d} S .
$$

where traction vector component $T_{i}$ is specified on the boundary subset $\Gamma_{T_{i}} \subset \Gamma$. Following the above authors, we include only quadratic terms in the gradients, but in a generalisation we also allow cross terms between $\nabla c$ and $\nabla \boldsymbol{e}$ in the non-uniform contribution $\mathscr{G}$, which can therefore be written as

$$
\begin{aligned}
\mathscr{G}(c, \boldsymbol{e}, \nabla c, \nabla \boldsymbol{e})= & \frac{1}{2} \nabla c \cdot \boldsymbol{\kappa}(c, \boldsymbol{e}) \nabla c+\sum_{\alpha, \beta} \frac{1}{2} \nabla e_{\alpha} \cdot \boldsymbol{\gamma}^{\alpha \beta}(c, \boldsymbol{e}) \nabla e_{\beta} \\
& +\sum_{\alpha} \nabla c \cdot \boldsymbol{\theta}^{\alpha}(c, \boldsymbol{e}) \nabla e_{\alpha} .
\end{aligned}
$$

Here $\boldsymbol{\kappa}$ is a symmetric tensor of composition-gradient energy coefficients, each $\gamma^{\alpha \beta}(\alpha, \beta=1, \ldots, 6)$ is a tensor of strain gradient energy coefficients, and each $\boldsymbol{\theta}^{\alpha}$ is a tensor of the mixed, composition-strain gradient energy coefficients. Note that, in general, these coefficients will be functions of the local composition and strain. The point group symmetry of the cubic reference crystal imposes constraints on the tensor components of $\boldsymbol{\kappa}, \boldsymbol{\gamma}^{\alpha \beta}$ and $\boldsymbol{\theta}^{\alpha}$ as well as on the form of $\mathscr{F}$.

Although the gradient energies bestow greater accuracy upon the free-energy description of solids with non-uniform composition and strain fields, they are essential at a more fundamental level if the homogeneous free-energy density is non-convex. At compositions that render $\mathscr{F}$ non-convex, the absence of a gradient energy term will allow spinodal decomposition characterised by composition fluctuations of arbitrary fineness, thus leading to non-unique microstructuresa fundamentally unphysical result. ${ }^{15}$ With $\boldsymbol{\kappa} \neq 0$, the compositiongradient energy penalises the interfaces wherein composition varies rapidly between high and low limits. This ensures physically realistic results, manifesting in unique microstructures with a mathematically well-posed formulation.

An essentially analogous situation exists with respect to the negative curvatures of $\mathscr{F}$ in the $e_{2}-e_{3}$ plane at low $c$, which drive the cubic lattice to distort into the tetragonal variants corresponding to the three free-energy wells. Consider a solid with a homogeneous free-energy density as in Figure 3 and a strain state lying between the valleys in the $e_{2}-e_{3}$ plane at $c=0$. Absent the strain gradient energy, mechanochemical spinodal decomposition would allow tetragonal variants of arbitrary fineness-an unphysical result, reflecting further mathematical ill-posedness. Retention of the strain gradient energy, $\left(\gamma^{\alpha \beta} \neq 0\right)$ penalises interfaces of sharply varying strain between tetragonal variants to ensure physically realistic results and unique microstructures from a mathematically well-posed formulation. This is well understood in the literature that studies the formation of martensitic microstructures from non-convex free-energy density functions. ${ }^{16-18}$

Governing equations of non-equilibrium chemistry. The free energy for non-homogeneous composition and strain fields (equation 2), must be a minimum at equilibrium. The state of a solid out of equilibrium will evolve to reduce the free energy $\Pi[c, u]$. In formulating a kinetic equation for the redistribution of atomic species through diffusion, we are guided by variational extremisation of the free energy to identify the chemical potential, $\mu$. Details of this calculation appear as Supplementary Information.
The result follows:

$$
\begin{aligned}
\mu= & \frac{\partial \mathscr{F}}{\partial c}-\nabla \cdot(\boldsymbol{\kappa} \nabla c)+\nabla c \cdot \frac{\partial \boldsymbol{\kappa}}{\partial c} \nabla c+\sum_{\alpha, \beta} \frac{1}{2} \nabla e_{a} \cdot \frac{\partial \boldsymbol{\gamma}^{\alpha \beta}}{\partial c} \nabla e_{\beta} \\
& +\sum_{a}\left(\nabla c \cdot \frac{\partial \boldsymbol{\theta}^{\alpha}}{\partial c} \nabla e_{\alpha}-\nabla \cdot\left(\boldsymbol{\theta}^{\alpha} \nabla e_{a}\right)\right) .
\end{aligned}
$$

For solids where $c$ tracks the composition of an interstitial element within a chemically inert host, such as $\mathrm{Li}$ in $\mathrm{Li}_{c} \mathrm{Mn}_{2} \mathrm{O}_{4}, \mu$ in equation (4) corresponds to the chemical potential of the interstitial element. If $c$ tracks the composition of a substitutional species, such as in alloys or on sublattices of complex compounds (e.g., the cation sublattice of yttria-stabilised zirconia), $\mu$ is equal to the chemical potential difference between the substitutional species.

The common phenomenological relation for the flux is $\boldsymbol{J}=-\boldsymbol{L}(\boldsymbol{c}, \boldsymbol{e}) \nabla \mu$, where $\boldsymbol{L}$ is the Onsager transport tensor (see de Groot and Mazur ${ }^{19}$ ). For an interstitial species, $\boldsymbol{L}$ is related to a mobility, ${ }^{20}$ whereas it is a kinetic, intermixing coefficient for a binary substitutional solid. ${ }^{21}$ Inserting the flux in a mass conservation equation yields the strong form of the governing partial differential equation for time-dependent mass transport. It is of fourth order in space due to the composition-gradient dependence of $\mu$ in equation (4). See Supplementary Information for strong and weak forms of this partial differential equation.

Governing equations of mechanical equilibrium: strain gradient elasticity. Mechanical equilibrium is assumed as elastic wave propagation typically is a much faster process than diffusional relaxation in crystalline solids. Equilibrium is imposed by extremising the free-energy functional with respect to the displacement field. Standard variational techniques lead to the weak and strong forms of strain gradient elasticity. The treatment is technical, for which reason we restrict ourselves to the constitutive relations that are counterparts to the chemical potential equation (4) for chemistry. Coordinate notation is used for transparency of the tensor algebra, and summation is implied over repeated spatial index, I. Details appear in Supplementary Information. The final form of the equations is complementary to that mentioned in Toupin, ${ }^{9}$ as our derivation is relative to the reference crystal, $\Omega$.

With the deformation gradient $\boldsymbol{F}$ being related to the GreenLagrange strain as $E_{K L}=\frac{1}{2}\left(F_{i K} F_{i L}-\delta_{K L}\right)$, the first Piola-Kirchhoff stress tensor and the higher-order stress tensor, respectively, are given by

$$
\begin{aligned}
& P_{i J}=\sum_{a} \frac{\partial(\mathscr{F}+\mathscr{G})}{\partial e_{a}} \frac{\partial e_{a}}{\partial F_{i J}}+\sum_{a} \frac{\partial \mathscr{G}}{\partial e_{a, l}} \frac{\partial e_{a, l}}{\partial F_{i J}} \\
& B_{i J K}=\sum_{a} \frac{\partial \mathscr{G}}{\partial e_{a, l}} \frac{\partial e_{a, l}}{\partial F_{i J, K}}
\end{aligned}
$$

The higher-order stress $\boldsymbol{B}$, which is absent in classical, non-gradient elasticity ${ }^{22}$ (and in earlier treatments of mechanochemistry ${ }^{23,24}$ ), makes the strong form of gradient elasticity a fourth order, nonlinear partial differential equation in space (Supplementary Information). The first three-dimensional solutions to general boundary value problems of Toupin's strain gradient elasticity theory at finite strains were recently presented by the authors. ${ }^{25}$

\section{RESULTS}

Two-dimensional examples

We first consider a two-dimensional solid to better visualise the microstructures that can emerge from mechanochemical spinodal decomposition. Plane strain elasticity is assumed, for which $E_{13}, E_{23}$ and $E_{33}=0$, giving $e_{4}$ and $e_{5}=0$, and $e_{3}=e_{1} / \sqrt{2}$, reducing 


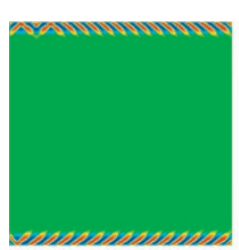

$\mathrm{t}=0.1$
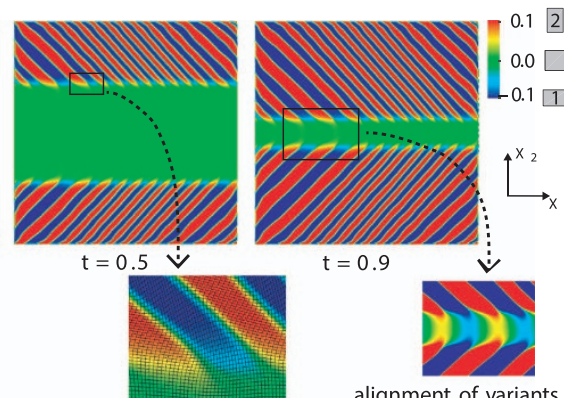

alignment of variants

mesh deformation

Figure 4. Evolution of 2D microstructure during outflux from the top and bottom surfaces of a solid under plane strain. Contours show strain $e_{2}$. Note the legend and corresponding square/rectangular variant crystal structures. The deformation and accompanying twinned microstructure are seen clearly in the distorted mesh.

equations (1, 2, 3, 4, 5 and 6) to two dimensions. The discussion on two-dimensional mechanochemical spinodal decomposition (Figure 2) holds: as a particular parameterisation of $\mathscr{F}\left(c, e_{1}, e_{2}, e_{6}\right)$, we consider a regular solution model as a function of $c$ at zero strain. At $c=0, \mathscr{F}\left(c, e_{1}, e_{2}, e_{6}\right)$ is double-welled in $e_{2}$, corresponding to the two rectangular variants. The gradient energy is $\mathscr{G}\left(\nabla c, \nabla e_{2}\right)$ with a constant isotropic $\boldsymbol{\kappa} \neq 0$ and constant $\gamma^{22} \neq 0$, all other gradient coefficients being zero. Although the fullest possible complexity of the coupling is not revealed by these simplifications, the aim here is to present the essential physics that is universal to mechanochemical spinodal decomposition, postponing details of the more complex couplings and tensorial forms to future communications, where they will be derived for specific materials systems. See Supplementary Information for specific forms of $\mathscr{F}\left(c, e_{1}, e_{2}, e_{6}\right)$ and $\mathscr{G}\left(\nabla c, \nabla e_{2}\right)$.

Figure 4 shows the evolution of microstructure over a $0.01 \times 0.01$ domain whose reference (initial) state has the square crystal structure and $c=1$. The displacement component $u_{1}=10^{-5}$ on the right boundary $\left(X_{1}=0.01\right)$, with the remaining boundaries fixed $(\boldsymbol{u}=0)$. An outward flux is imposed on the top and bottom boundaries causing a decrease in composition, $c$, starting from the boundaries. As the composition falls, the homogeneous free-energy density $\mathscr{F}$ loses convexity and the state of the material $\left(c, e_{2}\right)$ enters the mechanochemical spinodal along $e_{2}=0$ (Figure $2 \mathrm{~b}$ ). The continuing outward flux first drives material near the top and bottom boundaries fully into the regime, where the rectangular crystal structure is stable. As explained in Figure $2 b$, the negative curvature $\partial^{2} \mathscr{F} / \partial e_{2}^{2}<0$ creates thermodynamic driving forces that distort the square structure, shown in green, into rectangular variants, which form as red/blue laminae (all $e_{2}$ values). The coexistence of the parent, square structure with the daughter, rectangular variants also can give rise to cross-hatched microstructures that parallel the tweed microstructures described in the work of Karatha et al. ${ }^{13,14}$ Supplementary Movie S9 in the Supplementary Information shows the formation of such a microstructure.

A laminar, micro-twinned microstructure develops as the two rectangular variants form, distinguished by the sign of $e_{2}$ (see legend). Note that $e_{2}$ remains continuous because of the penalisation of strain gradients $\nabla e_{2}$, although discontinuities can develop in $\nabla e_{2}$ itself. Because our numerical framework uses basis functions that are continuous up to their first derivatives (see Methods and Supplementary Information), $\nabla e_{2}$ is indeed discontinuous in the computations. The lamination accommodates the strain difference between the two rectangular variants to minimise the free energy. If strains were infinitesimal $\left(\left|e_{1}\right|,\left|e_{2}\right|\right.$, $\left.\left|e_{6}\right| \gtrsim 0\right) e_{2}$ would correspond to shear along the directions that are rotated $\pi / 4$ radians from the crystal axes. But the strains in these microstructures can be finite (reflected in the range $-0.1 \leqslant e_{2} \leqslant 0.1$
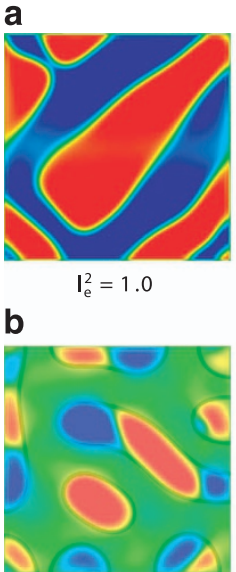

$\mathrm{I}_{\mathrm{e}}^{2}=1.0$

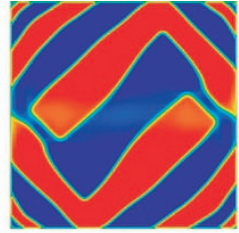

$\mathrm{I}_{\mathrm{e}}^{2}=0.1$

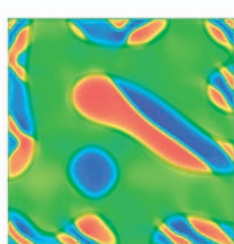

$\mathrm{I}_{\mathrm{e}}^{2}=0.1$
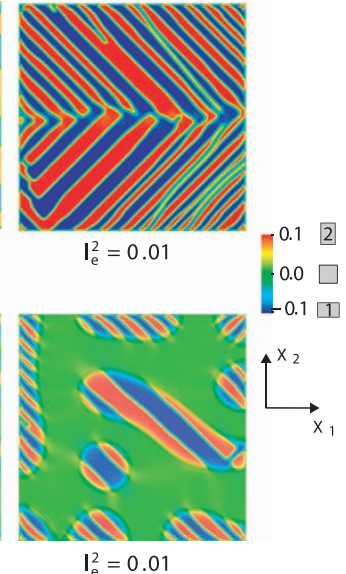

Figure 5. Microstructure controlled by elastic gradient length scale parameter $\left(l_{\mathrm{e}}\right)$. Shown are the final contours of $e_{2}$ for the simulation of (a) outflux from top and bottom surfaces, and (b) quenching.

in Figure 4) and involve large rotations to accommodate the rectangular variants; this necessitates a finite-strain formulation, as shown in the recent studies by Alphonse et $a l^{26}$ comparing infinitesimal-strain and finite-strain formulations for polytwinned microstructure evolution in martensitic alloys, and by Hildebrand and Miehe. ${ }^{7}$

The micro-twinning and coherency strains are seen in the distorted mesh of discretisation (inset of top centre panel). The undeformed mesh cells are squares, and hence the numerical discretisation strikingly delineates the kinematics of the cubic-torectangular transformation, and highlights the rectangular twins as well as the concentrated distortions along the twin boundaries. Supplementary Movie S6 in the Supplementary Information shows such mesh distortion and formation of the rectangular twins with a different form of the function $\mathscr{F}$.

In some cases, the long-range nature of elasticity forces like rectangular variants to align even when separated by an untransformed square phase. This is first seen in the finger-like extensions of strain contours from the rectangular variants into the square phase in Figure 4, followed by their alignment and eventual incorporation into laminae of the same variant (top right panel and its evolution shown as an inset). In this instance, although the micro-twins end at an invariant habit plane that is common with the untransformed square phase, the lattice parameters of the rectangular variants differ from those of the square phase, inducing elastic strain energy in the latter. The alignment lowers this strain energy, and also is seen in Figure 5a. Note, however, that this is not a universal feature. Supplementary Movie S2 shows instances where unlike variants come close to alignment.

The fineness of laminae depends on the strain gradient elasticity length scale $l_{\mathrm{e}} \sim \sqrt{\gamma^{22} / \sqrt{\sum_{a, \beta=1,2,6}\left(\partial^{2} \mathscr{F} / \partial e_{a} \partial e_{\beta}\right)^{2}}}$, as explored in Figure 5. In Figure 5a, the initial and boundary conditions are the same as in the example of Figure 4. Decreasing $l_{\mathrm{e}}$ weakens the penalty on the strain gradient $\nabla e_{2}$ across neighbouring, unlike rectangular variants and allows more twin boundaries. Notably, self-similarity is not maintained between microstructures for different $l_{\mathrm{e}}$, even for the same initial and boundary value problem. We understand this to be the influence of elastic strain accommodation: to minimise the total free energy when the strain gradient penalty changes, the physics optimises twin boundaries via laminations of different sizes as well as different patterns. Importantly, however, crystal symmetry admits non-vanishing strain gradient energy coefficients beyond $\gamma^{22} \neq 0$ 
used in these simulations (Supplementary Information). Furthermore, the composition dependence of $\mathscr{F}$ could be more complex than the simple regular solution model used here. Given the already strong effect of $l_{\mathrm{e}}$ alone, we conjecture that varying these forms will have a significant influence on the resulting coherent twin microstructure. The proper form, while guided by crystal symmetry arguments must ultimately be determined experimentally or by first-principles statistical mechanical methods.

The example in Figure $5 \mathrm{~b}$ further explores the influence of $I_{\mathrm{e}}$. In this suite of computations, the unstrained material with an initially square microstructure, convex free-energy density and composition having random fluctuations about $c=0.45$ was quenched to a low temperature and into the mechanochemical spinodal. The local composition and strain evolve under the thermodynamic driving forces detailed in the context of Figure 2. We draw attention, once again, to the changing identity of rectangular variants, their shapes and sizes depending on $l_{\mathrm{e}}$. Also, note the progressively finer lamination of rectangular domains with decreasing $l_{\mathrm{e}}$. Such studies suggest how dynamic mechanochemical spinodal decomposition can lead to an atlas of microstructures, which in turn will determine material properties. Supplementary Movies S1-S4 in the Supplementary Information show the evolution of some of these microstructures.

\section{A three-dimensional study}

This final example displays the full three-dimensional complexity of microstructures resulting from the mechanochemical spinodal. We persist with the above simplifications aimed at presenting the essential physics of mechanochemical spinodal decomposition, and postpone a full exploration of the coupling and anisotropies in coefficients to future work on specific materials systems. The free-energy density function used for the three-dimensional study appears as Supplementary Information.

Figure 6 shows the equilibrium microstructure that results in a solid that is initially in the cubic phase, $c=1$, subject to an outflux on all surfaces. The domain is a unit cube with displacement components $u_{2}, u_{3}=0.01$ on the boundary $X_{1}=1$, with zero displacement, $\boldsymbol{u}=0$, on the boundary $X_{1}=0$. The cubic-totetragonal transformation takes place as $c \rightarrow 0$. Under these conditions, all three tetragonal variants form, with an intricately interleaved microstructure for strain accommodation. Note the finer microstructures and changing pattern for smaller $l_{\mathrm{e}}$. The inset shows the surface straining around a corner, delineated by the distorted mesh lines. Observe the three, oriented, tetragonal variants formed by twinning deformation from the initial cubic structure. See Supplementary Movie S7 in the Supplementary Information for a detailed view of the three-dimensional structure

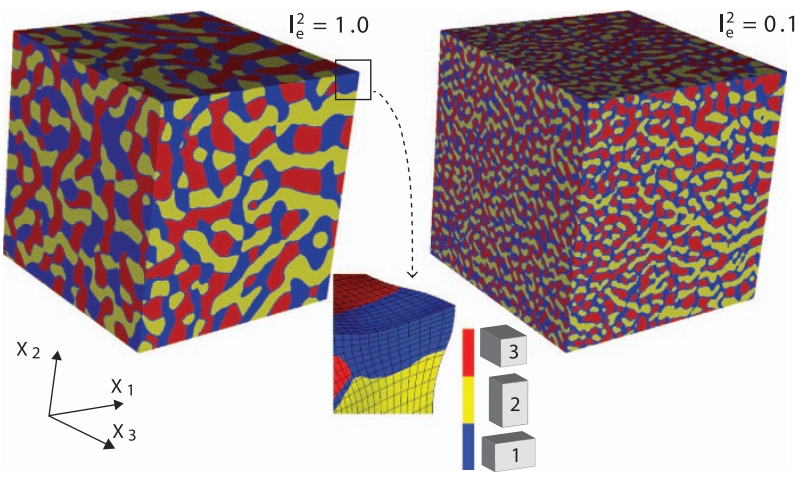

Figure 6. Microstructure observed in $3 D$ for different values of the elastic gradient length scale parameter $\left(I_{\mathrm{e}}\right)$. The three tetragonal variants appear in blue (variant 1 ), yellow (variant 2) and red (variant 3 ) for $c=0$. The transformation strains are easily discerned in the distorted mesh. of these individual tetragonal variants. Supplementary Movie S10 in Supplementary Information shows other three-dimensional microstructures whose cross-sectional planes bear closer resemblance to the plate-like structures predicted by twodimensional computations. To the best of our knowledge, such computations of a cubic-to-tetragonal transformation with twinned variants whose microstructure is controlled by nonlinear, strain gradient interface energies have not been previously presented.

\section{DISCUSSION}

Several kinetic mechanisms have been proposed to describe the decomposition of a solid upon entering a two-phase region. ${ }^{27,28}$ A commonly observed mechanism occurs through localised nucleation followed by diffusional growth that is mediated by the migration of interfaces separating the daughter phases from the parent phase. Nucleation and growth mechanisms are common when the phases participating in the transformation have little or no crystallographic relation to each other. The transformation then proceeds reconstructively, where the interphase interfaces are disordered or at best only semi-coherent. Numerical treatments of this mechanism rely on sharp-interface methods such as a level set approach. ${ }^{29}$

Other kinetic mechanisms of decomposition involving some degree of coherency are also possible when the participating phases share sufficient crystallographic commonality that order parameters can be defined describing continuous pathways connecting the parent and daughter phases. The structural changes of the crystal that accompany these transformations can fall in one of two categories. One subclass of structural transformations is driven by an internal shuffle, where the atomic arrangement within the unit cell of the crystal undergoes a symmetry-breaking change. The unit cell vectors of the crystal may also undergo a change and lower the symmetry of the lattice, but this effect is secondary and in response to the atomic shuffle within the unit cell. The order parameters for the structural change therefore describe the atomic shuffles within the unit cell and are non-conserved. The second subclass of structural transformations is driven by a symmetry-reducing change of the lattice vectors of the crystal, with any atomic rearrangement of the basis of the crystallographic unit cell occurring in response to the symmetrybreaking changes of the unit cell vectors. The natural order parameters describing these transitions are strains. The free energy in the first subclass will exhibit negative curvatures with respect to the non-conserved shuffle order parameters, whereas the free energy in the second subclass will have negative curvatures with respect to the relevant strain order parameters, as was recently predicted for the cubic-to-tetragonal transition of $\mathrm{ZrH}_{2}{ }^{3}$

Decomposition reactions that require both diffusional redistribution and a structural change due to an internal shuffle have been treated successfully and rigorously with phase field approaches that combine Cahn-Hilliard with Allen-Cahn. The Cahn-Hilliard description accounts for the composition degrees of freedom, whereas the Allen-Cahn component describes the evolution of the non-conserved shuffle order parameters required to distinguish the various phases of the transformation. ${ }^{4-7}$ These treatments have included strain energy as a secondary effect serving only as a positive contribution to the overall free energy due to coherency strains. The approach is rigorous if the free-energy density remains convex with respect to strain, with instabilities in the free energy appearing as a function of concentration and the non-conserved shuffle order parameters.

Here we have presented a mathematical formulation and an accompanying computational framework for decomposition reactions that combine diffusional redistribution with a structural change driven by a symmetry-breaking strain of the 
crystallographic unit cell as opposed to an internal shuffle within the unit cell. Strains have a primary role, explicitly serving as order parameters to distinguish variants of a daughter phase that has a symmetry subgroup-group relationship with its parent phase due to a structural change of the crystallographic unit cell. Crucial to the description is that the driving force for the formation of the daughter from a supersaturated parent phase emerges from an instability with respect to composition. The treatment therefore combines Cahn-Hilliard for composition with a description of martensitic transformations at fixed concentration introduced by Barsch and Krumhansl. ${ }^{8}$ The existence of simultaneous instabilities with respect to strain and composition has not been considered, as a mechanism to describe decomposition reactions upon quenching into a two-phase region.

The possibility of such a mechanochemical spinodal mechanism is motivated by recent first-principles studies of the cubic-totetragonal phase transformations of $\mathrm{ZrH}_{2}$, where the free energy of the high-temperature cubic form was predicted to become unstable with respect to strain upon cooling below the cubic-totetragonal second-order transition temperature. ${ }^{3}$ The $\mathrm{ZrH}_{2-2 c}$ hydride can accommodate large concentrations of hydrogen vacancies, $c$, and has a phase diagram that is topologically identical to that depicted in Figure $2 c$, with a two-phase region separating a hydrogen-rich tetragonal form of $\mathrm{ZrH}_{2-2 c a}$ from a cubic form of $\mathrm{ZrH}_{2-2 c \beta}$ (with $c_{a}<c_{\beta}$ ). See Zuzek et al. ${ }^{10}$ (in their phase diagrams, Zuzek et al. have an inverted composition axis relative to our notation, the tetragonal phase is labelled $\varepsilon$ and the cubic phase is $\delta$ ). To be consistent with the predicted free energies for stoichiometric $\mathrm{ZrH}_{2}$ (i.e., $c=0$ ) and the experimental $T$ versus $c$ phase diagram with the form of Figure 2c, the free energy of this hydride as a function of composition and strain (i.e., $e_{2}$ and $e_{3}$ ) should be similar to those depicted in Figures $2 \mathrm{a}, \mathrm{b}$ and 3 . Decomposition upon quenching cubic $\mathrm{ZrH}_{2-2 c}$ into the two-phase region can therefore proceed through a mechanochemical spinodal decomposition mechanism.

Not only does the phenomenological description introduced in this work describe a new mechanism of decomposition that is qualitatively distinct from previous combined Cahn-Hilliard and Allen-Cahn approaches, its numerical solution also proves to be substantially more complex due to contributions from strain gradient terms. Indeed, even numerical solutions to general, three-dimensional boundary value problems of gradient elasticity at finite strain were not available until presented recently by the authors. ${ }^{25}$ Furthermore, as other authors have pointed out before, the use of strain metrics as order parameters makes a reliance on infinitesimal strains untenable due to the rigid rotations that accompany the finite strains characterising most structural transformations. ${ }^{7,26}$ The use of finite-strain metrics introduces geometric nonlinearity into the problem, which although having been treated in past Cahn-Hilliard and Allen-Cahn approaches, presents additional numerical challenges when also considering strain gradient contributions. These challenges have been overcome in this work as demonstrated by our three-dimensional numerical examples.

Mechanochemical spinodal decomposition as described here can be expected in solids forming high-temperature phases that exhibit dynamical instabilities at low temperature. An accumulating body of first-principles calculations of the Born-Oppenheimer surfaces have shown that many high-temperature phases are dynamically unstable at low temperature, ${ }^{30,31}$ becoming stable at high temperature through large anharmonic vibrational excitations, ${ }^{3,32-35}$ usually through a second-order phase transition. Although such instabilities are frequently dominated by phonon modes describing an internal shuffle, a subset of chemistries become dynamically unstable at low temperature with respect to phonon modes that break the symmetry of the crystal unit cell, 3,34 an instability that can be described phenomenologically with strain order parameters. If, upon alloying such compounds, the high-symmetry phase becomes stable by passing through a two-phase region, its free-energy surface will resemble that of Figures $2 \mathrm{~b}$ and 3 , and thereby make the solid susceptible to the mechanochemical spinodal decomposition mechanism described here.

Although first-principles and experimental evidence suggest that mechanochemical spinodal decomposition should occur in $\mathrm{ZrH}_{2-2 c}$, we expect it to occur in a wide range of other chemistries as well. One possible example, as described in the Introduction, is the decomposition of cubic yttria stabilised zirconia ${ }^{36,37}$ upon quenching from the high-temperature cubic phase into a two-phase region separating a low-Y tetragonal phase from a Y-rich cubic phase. Past treatments of this transformation ${ }^{4-6}$ relied on non-conserved order parameters to distinguish the different tetragonal variants from each other and from the cubic parent phase. The elastic part of the free-energy density function was parameterised using linearised elasticity and infinitesimal strains, as other authors have also done. ${ }^{38,39}$ The chemical part of the free-energy density was assumed to have a negative curvature as a function of the non-conserved order parameter at Zr-rich compositions, but made up of convex (and quadratic) potentials with respect to strain. We reiterate that such a treatment rests on the implicit assumption that internal shuffles drive the cubic-to-tetragonal transformation, whereas the free energy as a function of strain remains convex for all relevant values of the non-conserved order parameters.

The possibility also exists that a coarse-grained free-energy density of cubic $\mathrm{ZrO}_{2}$ exhibits negative curvatures with respect to strain below the cubic-to-tetragonal transition temperature making the formalism developed here an accurate description of decomposition reactions of yttria-stabilised zirconia. Although the precise mechanism and nature of the cubic-to-tetragonal transition of pure $\mathrm{ZrO}_{2}$ remain to be resolved, ${ }^{33}$ first-principles calculations predict that the cubic form of $\mathrm{ZrO}_{2}$ is dynamically unstable with respect to the transformation to the tetragonal variant. ${ }^{40}$ A rigorous statistical mechanics treatment ${ }^{3,32}$ is required to determine whether the cubic-to-tetragonal transition of pure $\mathrm{ZrO}_{2}$ is accompanied by a change in the sign of the curvature of the free-energy density with respect to $e_{2}$ and $e_{3}$. If this proves to be the case, yttria-stabilised zirconia should also be susceptible to the mechanochemical spinodal decomposition upon quenching, consistent with the coherent spinodal microstructures between tetragonal and cubic phases observed in single-crystal regions of quenched cubic $\mathrm{Zr}_{1}-{ }_{c} \mathrm{Y}_{x} \mathrm{O}_{2-c / 2}{ }^{37}$

A mechanochemical spinodal may also have a role in a variety of important electrode materials for Li-ion batteries, and intercalation compounds considered for two-dimensional nanoelectronics. These include cubic $\mathrm{LiMn}_{2} \mathrm{O}_{4}$ transforming to tetragonal $\mathrm{Li}_{2} \mathrm{Mn}_{2} \mathrm{O}_{4}{ }^{41}$ Although most mechanochemical spinodal transitions will occur in three dimensions, many of the qualitative features of these transitions are more conveniently illustrated in two dimensions. Our two-dimensional studies should also prove relevant to understanding mechanochemical phase transformations in two-dimensional layered materials for nanoelectronics. Materials such as $\mathrm{TaS}_{2}$, are susceptible to Peierls instabilities upon variation of the composition of adsorbed or intercalated guest species that donate to or extract electrons from the sheet-like host. ${ }^{42}$ Our phenomenological treatment by introduction of the concept of a mechanochemical spinodal, coupled with gradient stabilisation of the ensuing non-convex free energy in straincomposition space, thus offers a framework with potential for extension to a wide range of phase transformation phenomena.

With regard to the fundamental thermodynamic underpinnings of analogous processes, the phenomenological model introduced here can also be used to describe temperature-driven martensitic transformations. The composition, $c$, would then be analogous to the internal energy density, $\mathscr{U}$, whereas the chemical potential, $\mu$, would be analogous to the temperature, $T$. Due to the presence 
of temperature gradients throughout the solid, though, the starting point must be a formulation of the entropy density, $\mathscr{S}$, (as opposed to the Helmholtz free energy) as a functional of spatially varying $\mathscr{U}$ and displacements, $\boldsymbol{u}$. In analogy with equation (2), the total entropy can be written as a volume integral over a homogeneous entropy density that depends on the local internal energy density and strain $\overline{\mathscr{S}}(\mathscr{U}, e)$, as well as a non-uniform entropy density contribution expressed in terms of gradients in internal energy density and strain $(\nabla \mathscr{U}, \nabla \boldsymbol{e})$. Variational maximisation of the entropy will yield mechanical equilibrium equations as well as an expression for the temperature $T$ (strictly speaking, for its reciprocal, $1 / T$ ), similar to equation (4) for the chemical potential. Due to the presence of gradient terms, $\nabla \mathscr{U}$ and $\nabla \boldsymbol{e}$, the temperature will not only be a function of the local internal energy density and strain, but also gradients in these field variables. As with the diffusion problem treated here, heat flow can be described with an Onsager expression relating the heat flux to a gradient in temperature. The possibility exists that the homogeneous entropy $\overline{\mathscr{S}}$ exhibits instabilities with respect to internal energy density $\mathscr{U}$, allowing for spinodal decomposition with respect to the redistribution of $\mathscr{U}$ in a manner that is similar to the well understood problem of spinodal decomposition with respect to composition. The treatment introduced here can therefore describe (upon replacing $c$ with $\mathscr{U}$ and $\mu$ with $T$ ) temperature-driven martensitic transformations for solids that exhibit instabilities with respect to both internal energy density and strain. Past treatments of this phenomenon also relied on a Barsch and Krumhansl approach, solving the mechanical problem together with Fourier's law of heat conduction. ${ }^{38,39}$ However, these studies were restricted to two dimensions, ${ }^{26,38,39}$ with some neglecting geometric nonlinearity by relying on infinitesimal strains. ${ }^{38,39}$ At a more fundamental level, they did not consider the possibility of spinodal instabilities with respect to the redistribution of internal energy density.

\section{MATERIALS AND METHODS}

The governing fourth-order partial differential equations of mass transport and gradient elasticity are solved numerically in weak form, wherein second-order spatial gradients appear on the trial solutions and their variations. Numerical solutions require basis functions that are continuous up to their first spatial gradients, at least. Our numerical framework (see Rudraraju et al. ${ }^{25}$ ) uses isogeometric analysis ${ }^{43}$ and the PetIGA code framework $^{44}$ with spline basis functions, which can be constructed for arbitrary degree of continuity (Supplementary Information). This framework has proven pivotal to the current work. The code used for the numerical examples in this paper can be downloaded at the University of Michigan Computational Physics Group open-source codes webpage: http://www.umich.edu/ compphys/codes.html

\section{ACKNOWLEDGEMENTS}

The mathematical formulation for this work was carried out under an NSF CDI Type I grant: CHE1027729 'Meta-Codes for Computational Kinetics' and NSF DMR 1105672. The numerical formulation and computations have been carried out as part of research supported by the US Department of Energy, Office of Basic Energy Sciences, Division of Materials Sciences and Engineering under award no. DE-SC0008637 that funds the PRedictive Integrated Structural Materials Science (PRISMS) Center at University of Michigan.

\section{COMPETING INTERESTS}

The authors declare no conflict of interest.

\section{REFERENCES}

1. Cahn, J. W. \& Hilliard, J. E. Free energy of a nonuniform system. i interfacial energy. J. Chem. Phys. 28, 258-267 (1958).

2. Allen, S. M. \& Cahn, J. W. A microscopic theory for antiphase boundary motion and its application to antiphase boundary coarsening. Acta Metallurgica 27, 1085-1091 (1979).
3. Thomas, J. C. \& Van der Ven, A. Finite-temperature properties of strongly anharmonic and mechanically unstable crystal phases from first principles. Phys. Rev. B 88, 214111 (2013).

4. Wang, Y., Wang, H., Chen, L. Q. \& Khatchaturyan, A. G. Shape evolution of a coherent tetragonal precipitate in partially stabilized cubic zirconia: a computer simulation. J. Am. Ceram. Soc. 76, 3029-3033 (1993).

5. Fan, D. \& Chen, L. Q. Computer simulation of twin formation during the displacive $c \rightarrow t^{\prime}$ phase transformation in the Zirconia-Yttria system. J. Am. Ceram. Soc. 78, 769-773 (1995).

6. Fan, D. \& Chen, L. Q. Possibility of spinodal decomposition in $\mathrm{ZrO}_{2}-\mathrm{Y}_{2} \mathrm{O}_{3}$ alloys: a theoretical investigation. J. Am. Ceram. Soc. 78, 1680-1686 (1995).

7. Hildebrand, F. E. \& Miehe, C. A phase field model for the formation and evolution of martensitic laminate microstructure at finite strains. Philos. Mag. 92, 4250-4290 (2012).

8. Barsch, G. R. \& Krumhansl, J. A. Twin boundaries in ferroelastic media without interface dislocations. Phys. Rev. Lett. 53, 1069-1072 (1984).

9. Toupin, R. A. Elastic materials with couple-stresses. Arch. Ration. Mech. Anal. 11, 385-414 (1962).

10. Zuzek, E., Abriata, J. P., San-Martin, A. \& Manchester, F. D. The H-Zr (hydrogenzirconium) system. Bull. Alloy Phase Diagr. 11, 385 (1990).

11. Bhattacharya, K., Conti, S., Zanzotto, G. \& Zimmer, J. Crystal symmetry and the reversibility of martensitic transformations. Nature 428, 55-59 (2004).

12. van der Waals, J. D. Thermodynamische theorie der capillariteit in onderstelling van continue dichtheidsverandering. Verhanderlingen der Koninklijke Nederlandse Akademie 1, 3-56 (1893).

13. Kartha, S., Castan, T., Krumhansl, J. A. \& Sethna, J. P. Spin-glass nature of tweed precursors in martensitic transformations. Phys. Rev. Lett. 67, 3630-3633 (1991).

14. Kartha, S., Krumhansl, J. A., Sethna, J. P. \& Wickham, L. K. Disorder-driven pretransitional tweed pattern in martensitic transformations. Phys. Rev. B 52, 803-827 (1995).

15. Hilliard, J. E. in Phase Transformations (ed Aronson, H. I.) 470-560 (ASM, 1970).

16. Ball, J. M. \& James, R. D. Fine phase mixtures as minimizers of energy. Arch. Ration. Mech. Anal. 100, 13-52 (1987).

17. Müller, S. Calculus of Variations and Geometric Evolution Problems (Cetraro, 1996). Lecture Notes in Mathematics, vol. 1713 (Springer, 1999).

18. Ball, J. M. \& Crooks, E. C. M. Local minimizers and planar interfaces in a phase-transition model with interfacial energy. Calc. Var. Partial. Differ. Equ. 40, 501-538 (2011)

19. de Groot, S. R. \& Mazur, P. Non-Equilibrium Thermodynamics (Dover, 1984).

20. Van der Ven, A., Bhattacharya, J \& Belak, A. A. Understanding li diffusion in li-intercalation compounds. Acc. Chem. Res. 46, 1216-1225 (2013).

21. Van der Ven, A., Yu, H. C., Ceder, G. \& Thornton, K. Vacancy mediated substitutional diffusion in binary crystalline solids. Prog. Mater. Sci. 55, 61-105 (2010).

22. Truesdell, C. \& Noll, W. The Nonlinear Field Theories of Mechanics (Springer Verlag, 1965).

23. Voorhees, P. W. \& Johnson, W. C. The thermodynamics of elastically stressed crystals. Solid State Phys. Adv. Res. Appl. 59, 1-201 (2004).

24. Larche, F. \& Cahn, J. W. Non-linear theory of thermochemical equilibrium of solids under stress. Acta Metallurgica 26, 53-60 (1978).

25. Rudraraju, S., Van der Ven, A. \& Garikipati, K. Three-dimensional isogeometric solutions to general boundary value problems of Toupin's gradient elasticity theory at finite strains. Comput. Methods Appl. Mech. Eng. 278, 705-728 (2014).

26. Alphonse, F., Le Bouar, Y., Gaubert, A. \& Salman, U. Phase field methods: microstructures, mechanical properties and complexity. Comptes Rendus Physique 11, 245-256 (2010).

27. Balluffi, R. W., Allen, A. M. \& Carter, W. C. Kinetics of Materials (John Wiley \& Sons, 2005).

28. Porter, D. A. \& Easterling, K. E. Phase transformations in metals and alloys (Nelson Thorne, Ltd., 2001).

29. Sethian, J. A. Level Set Methods and Fast Marching Methods: Evolving Interfaces in Computational Geometry, Fluid Mechanics, Computer Vision and Materials Science. (Cambridge Univ. Press, 1999).

30. Craievich, P. J., Weinert, M., Sanchez, J. M. \& Watson, R. E. Local stability of nonequilibrium phases. Phys. Rev. Lett. 72, 3076-3079 (1994).

31. Grimvall, G., Magyair-Koepe, B., Ozolins, V. \& Persson, K. A. Lattice instabilities in metallic elements. Rev. Mod. Phys. 84, 945-986 (2012).

32. Zhong, W., Vanderbilt, D. \& Rabe, K. A. Phase transitions in $\mathrm{BaTiO}_{3}$ from first-principles. Phys. Rev. Lett. 73, 1861-1864 (1994).

33. Fabris, S., Paxton, A. T. \& Finnis, M. W. Free energy and molecular dynamics calculations for the cubic-tetragonal phase transition in zirconia. Phys. Rev. B 63, 094101 (2001)

34. Bhattacharya, J. \& Van der Ven, A. Mechanical instabilities and structural phase transitions: The cubic to tetragonal transformation. Acta Materiala 56, 4226-4232 (2008) 
35. Souvatzis, P., Eriksson, O., Katsnelson, M. I. \& Rudin, S. P. Entropy driven stabilization of energetically unstable crystal structures explained from first principles theory. Phys. Rev. Lett. 100, 095901 (2008).

36. Chien, F. R., Ubic, F. J., Prakash, V. \& Heuer, A. H. Stress-induced martensitic transformation and ferroelastic deformation adjacent microhardness indents in tetragonal zirconia single crystals. Acta Materialia 46, 2151-2171 (1998).

37. Krogstad, J. A. et al. Phase stability of t'-Zirconia-based thermal barrier coatings: mechanistic insights. J. Am. Ceram. Soc. 94, S168-S177 (2011).

38. Bouville, M. \& Ahluwalia, R. Effect of lattice-mismatch-induced strains on coupled diffusive and displacive phase transformations. Phys. Rev. B 75, 054110 (2007).

39. Maraldi, M., Wells, G. \& Molari, L. Phase field model for coupled displacive and diffusive microstructural processes under thermal loading. J. Mech. Phys. Solids 59, 1596-1612 (2011)

40. Jomard., G. et al. First-principles calculations to describe zirconia pseudopolymorphs. Phys. Rev. B 59, 4044-4052 (1999).

41. Thackeray, M. M. Manganese oxides for lithium batteries. Prog. Solid State Chem. 25, 1-71 (1997).
42. Rossnagel, K. Suppression and emergence of charge-density waves at the surfaces of layered $1 T-\mathrm{TiSe}_{2}$ and $1 \mathrm{~T}-\mathrm{TaS}_{2}$ by in situ Rb deposition. New J. Phys. 12, 125018 (2010).

43. Cottrell, J., Hughes, T. J .R. \& Bazilevs, Y. Isogeometric Analysis: Toward Integration of CAD and FEA (Wiley, 2009).

44. Collier, N., Dalcín, L. \& Calo, L. M. Petiga: high-performance isogeometric analysis. Preprint at http://arXiv:1305.4452 (2013).

(c) This work is licensed under a Creative Commons Attribution 4.0 International License. The images or other third party material in this article are included in the article's Creative Commons license, unless indicated otherwise in the credit line; if the material is not included under the Creative Commons license, users will need to obtain permission from the license holder to reproduce the material. To view a copy of this license, visit http://creativecommons.org/licenses/ by/4.0/

(C) The Author(s) 2016

Supplementary Information accompanies the paper on the npj Computational Materials website (http://www.nature.com/npjcompumats) 